\title{
Diversité et composition phytochimique des bâtonnets frotte-dents (cure-dents) proposés chez les Peul de la commune de Tessékéré (Ferlo Nord, Sénégal)
}

\author{
Bétémondji Désiré Diatta ${ }^{*}$, Ousmane Niass ${ }^{2}$, Massamba Diouf $^{3}$, Mathieu Guèye ${ }^{4}$, Emeline Houel$^{5}$, \\ Gilles Boetsch 6 \\ 1 Doctorant à l'UMI 3189 « Environnement, Santé et Société », Faculté de Médecine Pharmacie Odontostomatologie \\ UCAD, Sénégal, betemondjidesire@hotmail.fr \\ 2 Laboratoire de chimie analytique Faculté de Médecine Pharmacie Odontostomatologie à l'université Cheikh Anta \\ Diop de Dakar, Afrique \\ ${ }^{3} \mathrm{PhD}$ au laboratoire d'Épidémiologie et santé publique Université Cheikh Anta Diop / UMI 3189 / CNRS, Sénégal \\ ${ }^{4}$ Département de Botanique et Géologie, Laboratoire de Botanique, IFAN Cheikh. A. Diop/UCAD BP 206 Dakar ; UMI \\ 3189, Sénégal \\ ${ }^{5}$ CNRS, UMR EcoFoG, AgroParisTech, Cirad, INRA, Université des Antilles, Université de Guyane, 97300 Cayenne, \\ France \\ ${ }^{6}$ Directeur de recherche au CNRS, UMI 3189 Environnement, santé société, faculté de médecine secteur Nord, \\ Marseille, France e-mail : betemondjidesire@hotmail.fr
}

Original submitted in on $4^{\text {th }}$ December 2020. Published online at www.m.elewa.org/journals/ on $28^{\text {th }}$ February 2021 https://doi.org/10.35759/JABs.158.1

\section{RÉSUMÉ}

Objectifs : Le but de cette étude était de déterminer la composition phytochimique et la teneur en grandes classes chimiques des tiges les plus utilisées comme bâtonnets frotte-dents chez les peul de Widou Thiengoli au Nord Ferlo (Sénégal).

Méthodes et résultats : Des entretiens ouverts semi structurés ont permis de recueillir les plantes les plus utilisées. L'indice de fidélité renseigne sur la convergence des usages quant à l'emploi des plantes comme bâtonnets frotte-dent comparé aux autres pratiques cosmétiques. L'étude de la composition phytochimique des tiges a ciblé 8 classes chimiques. Les tanins et les saponines sont très fréquents ; les terpénoïdes et les leuco-anthocyanines presque inexistants. Un dosage des flavonoïdes, alcaloïdes et polyphénols, réalisé chez des extraits aqueux, a porté sur 12 plantes. Les meilleures teneurs en polyphénols et alcaloïdes sont recueillies chez Anogeissus leiocarpa, et la meilleure en flavonoïdes chez Commiphora africana.

Conclusion et applicabilité des résultats : Ces résultats permettent de sélectionner à travers la composition phytochimique des plantes, les espèces présentant de potentielles activités antimicrobiennes, car renfermant des composés phytochimique doués de fonctions germicides, au- delà de la fonction mécanique connue des bâtonnets dans l'élimination de la plaque dentaire.

Mots clés : Bâtonnets frotte-dents, Dosage phytochimique, Ethnobotanique, Téssékéré, Ferlo Nord, 52 Sénégal 
Diversity and phytochemical composition of plants used as toothbrush sticks (toothpicks) by Fulani of Tessékéré commune (North Ferlo, Senegal)

\section{ABSTRACT}

Objectives: The aim of this study was to determine the phytochemical composition and the content of major chemical classes of the stems most used as tooth-brushing sticks among the Fulani of Widou Thiengoli in North Ferlo (Senegal).

Methods and results: Open semi-structured interviews made it possible to collect data on the most used plants. The fidelity index provides information on the convergence of uses regarding the use of plants as toothbrushes compared to other cosmetic practices. The study of the phytochemical composition of the stems targeted 8 chemical classes. Tannins and saponins were very common; almost non- existent were the terpenoids and leuco-anthocyanins. An assay of flavonoids, alkaloids and polypnenols, in aqueous extracts, was carried out on 12 plants. The best contents of polyphenols and alkaloids were obtained from Anogeissus leiocarpa, and the best of flavonoids from Commiphora africana.

Conclusion and applicability of the results: These results make it possible to select, through the phytochemical composition of plants, the species exhibiting potential antimicrobial activities, because they contain phytochemical compounds endowed with germicidal functions, beyond the known mechanical function of the rods in the plan removal of dental plaque.

Keywords: Toothbrush sticks, Phytochemical determination, Ethnobotany, Téssékéré, North Ferlo, and Senegal.

\section{INTRODUCTION}

Les populations peules du Ferlo sont tenues d'affronter à la fois une détérioration de leur écosystème, en particulier en termes de biodiversité mais, également à une mutation des habitudes et pratiques quotidiennes (Diallo et al., 2012 ; Ngom et al., 2013 ; Niang et al., 2014). La santé buccodentaire est un des aspects impactés par ces mutations, avec notamment l'observation d'une recrudescence des pathologies carieuses et parodontales liée à un changement des modes de vie et d'alimentation (Diouf et al., 2013). Ainsi, le projet africain de la Grande Muraille Verte (GMV) a décidé de rétablir la flore du sahel sénégalais à travers une sélection dans les parcelles de reboisement d'essences végétales connues des populations autochtones (Guissé, 2013). Le choix des plantes n'est pas anodin, il prend en compte les relations hommes- milieux suivant une démarche fortement recommandée dans le cadre de ce projet pour son appropriation par les populations locales (Guissé, 2013). Les plantes utilisées par ces populations comme bâtonnets frotte-dents pour prévenir les parodontopathies et les caries n'ont pas été omis dans cette sélection. La carie dentaire, apparue au Néolithique il y a environ 7000 ans avec la consommation des farines produites grâce à la culture des céréales selon Pasquini (2013), marque en réalité le passage des populations jusque-là nomades à un mode de vie sédentaire (Janot, 2013). La même évolution semble avoir eu lieu au Ferlo depuis la fabrication des forages dans une initiative de fixer ces populations jusque-là transhumantes (Ancey et al., 2009), transformant ainsi leurs comportements alimentaires (Diatta et al., 2019). L'implantation des forages a par exemple favorisé la création des marchés hebdomadaires (Guibert, 2008), aboutissant à la consommation de plus de produits transformés, surtout sucrés. La consommation au Ferlo de produits d'addiction (tabac et alcool) au-delà d'une certaine limite, liée à la forte boisson de lait et de thé (produits alimentaires positivement corrélés au CPITN), paramètre estimant les besoins de traitements parodontaux d'une communauté) expliquent la dégradation de la santé orale (Diouf et al., 2013 ; Diatta et al., 2019). Ces aliments forment sur les surfaces dentaires de la plaque qui peut se calcifier dans le temps et devenir du tarte. Une action mécanique d'une brosse à dent ou d'un bâtonnet frotte-dents peut empêcher la formation de ces dépôts calcifiés (Ajay et al., 2011). Chez les populations peules du Ferlo, les bâtonnets frotte- 
dents communément appelés cure-85 dents, sont plus souvent utilisés pour éliminer la plaque dentaire. Ces matériels d'hygiène orale sont 86 aussi retrouvés dans d'autres communautés africaines ou ailleurs dans le monde (Pasquini, 2013). Ces bâtons à mâcher sont des produits forestiers secondaires utilisés par des personnes de 88 tous âges (Lewis, 1980). Leur utilité selon les propriétés pharmacologiques de l'espèce (Khan et al., 2000; Al Lafi et Ababneh, 1995) et leur importance selon les représentations sociales (Blay, 2004 ; Arbonier, 2000), sont plus ou moins étudiées. Les cure-dents sont en effet très accessibles, peu coûteux et culturellement acceptables dans de nombreux pays en développement (Khan et al., 2000). Suivant ce qui précède et au regard du rôle mécanique de nettoyage des dents, il reste à savoir quelles espèces végétales privilégier pour une meilleure prévention. Certains composés chimiques de plantes sont reconnus pour leurs effets

\section{MATÉRIEL ET MÉTHODE}

Cadre d'étude L'étude a été réalisée dans la commune de Tessékéré au Ferlo Nord (Fig. 1). Le Ferlo couvre une superficie de $75000 \mathrm{~km}^{2}$. II se localise entre la vallée du fleuve Sénégal (au Nord et à l'Est), le lac de Guiers (à l'Ouest) et la vallée fossile du Ferlo (au Sud) (Diatta et al., 2019). II présente les principaux traits climatiques de la bande sahélienne. Ce climat se caractérise en effet par une pluviométrie moyenne comprise entre 200 et antibactériens (alcaloïdes, tannins, flavonoïdes, huiles essentielles), anti-inflammatoires (alcaloïdes, flavonoïdes, saponines, huiles essentielles, stéroïdes et terpénoïdes), analgésiques (saponines, stéroïdes et terpénoïdes), anesthésiques (alcaloïdes), antiseptiques (tanins, huiles essentielles), anti-œdème (saponines) (Weller, 1998 ; Houghton et Rahman, 1998). Des alcaloïdes isolés de Mahonia aquifolium exercent une activité antimicrobienne contre des bactéries Gram positives et Gram-négatives, des champignons et des protozoaires (Rohrer et Kunz, 2007). Ainsi, l'objectif de cette étude était d'identifier les plantes les plus utilisées comme cure-dents et de déterminer leur composition phytochimique en recensant les grandes classes chimiques (polyphénols, alcaloïdes, flavonoïdes) présentes afin d'orienter les populations vers celles qui présenteraient de potentielles fortes activités antimicrobiennes vis-à-vis des agents cariogènes ou parodontopathogènes.

$400 \mathrm{~mm}$. C'est le climat sahélien type (Aubreville, 1949) où l'année est divisée communément en deux saisons : une saison des pluies de juillet à octobre assez variable avec l'avènement de la sécheresse, donc instable ; une saison sèche qui dure 8 à 9 mois, de novembre à juin (Bakhoum, 2013). Le climat du Ferlo se distingue par une pluviométrie annuelle moyenne tournant entre 300 et $600 \mathrm{~mm}$ (CSE, 2007).

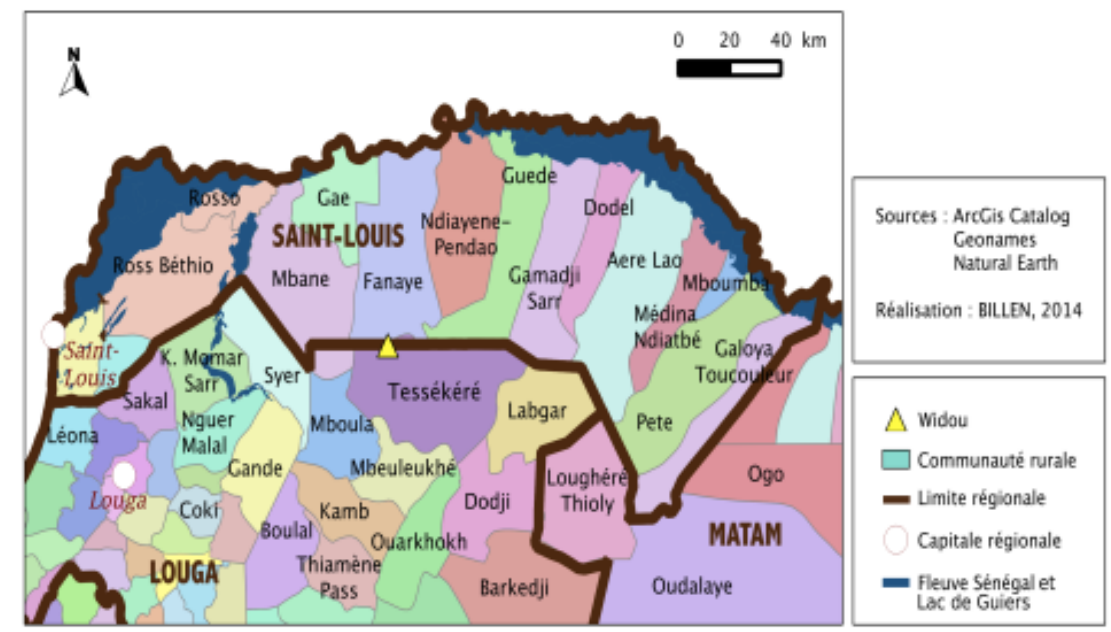

Fig. 1 : Carte des communes et des régions du Ferlo centrée sur Widou (Commune de Tessékéré) 
Recueil des données ethnobotaniques Le recensement des plantes utilisées comme bâtonnets frotte-dents et la perception locale de la pratique ont été évalués au moyen d'enquêtes ethnobotaniques (Weller, 1998 ; Heinrich et al., 2009 ; Weckerle et al., 2018). Les enquêtes ont été faites grâce à un guide d'entretien élaboré de manière à recueillir tous les noms des plantes ayant une ou des fonctions cosmétiques dans la zone, les parties utilisées, le mode de préparation du produit, le mode d'utilisation et la partie du corps ciblée. Les espèces proposées comme bâtonnets frotte-dents pour l'hygiène bucco-dentaire sont ici analysées. Les enquêtes ethnobotaniques ont été réalisées au cours de l'année 2014 et au cours de trois missions de terrain dont deux (2) en saison sèche (mars, avril, mai) et une en saison des pluies (septembre). Nous avons centré l'approche de notre étude sur les relations homme/milieu. L'âge minimal des enquêtés a été limité à 15 ans, âge requis pour disposer d'une certaine maturité physique et culturelle (Ohmagari et Berkes, 1997 ; Hun, 2002 ; Zarger, 2002). L'enquête a ciblé plus particulièrement les femmes âgées, potentiellement détentrices d'informations relatives aux savoirs et savoirfaire cosmétiques traditionnels. Les connaissances des jeunes femmes et de certains hommes ont aussi été évaluées, l'âge minimal étant limité à 15 ans. Toutes les plantes citées et existantes dans la zone ont été récoltées et mises en herbier. Des cure-dents ou bâtonnets frotte-dents de chaque espèce ont aussi été rapportés. Pour chaque plante récoltée, le lieu de récolte, le nom scientifique et le nom en Peul et les usages ont été indiqués. L'herborisation est faite de préférence en recueillant des échantillons fertiles comportant des feuilles, fleurs et des fruits. Ceci permet d'éviter les confusions possibles liées au fait que les plantes du Ferlo, soumises à divers stress, présentent souvent des formes différentes de celles de la même espèce vivant dans des milieux plus propices. Les tiges ont été récoltées en saison sèches, période pendant laquelle la conservation et le séchage sont plus faciles. Pour estimer le degré de popularité de chaque plante utilisée comme cure-dents, l'indice de fidélité (IF) a été calculé selon la méthode de Begossi (1996) suivante : IF $=$ Ip / lu $\times 100$ lp est le nombre d'enquêtés reconnaissant l'emploi d'une espèce précise dans une catégorie d'usage donnée et lu est le nombre total d'informateurs ayant proposé l'espèce dans toutes les catégories d'usages.

\section{Analyse phytochimique}

Extraction Les bâtonnets frotte-dents récoltés sont séchés à l'ombre puis broyés en fine poudre. Les extraits ont donc été préparés à l'eau car pour évaluer une activité biologique liée à un usage traditionnel, il est important de rester au plus proche de cet usage. Ce protocole a déjà été employé par différents auteurs lors de l'étude de l'activité biologique de bâtonnets frottedents (Rotimi et al., 1998 ; AbdelRahmane et al., 2002). Une prise d'essai $15 \mathrm{~g}$ de poudre de chaque échantillon de plante est mise à infuser avec $150 \mathrm{ml}$ d'eau à $90^{\circ} \mathrm{C}$ jusqu'au refroidissement. Le mélange ainsi obtenu est filtré sous vide et la partie aqueuse est concentrée à sec à l'évaporateur rotatif. Les extraits aqueux obtenus sont utilisés pour évaluer la composition phytochimique et doser les alcaloïdes, les flavonoïdes et les polyphénols.

Détermination de la composition phytochimique

Détermination des stéroïdes Un millilitre d'infusé aqueux a été dissout dans $10 \mathrm{ml}$ de chloroforme et un volume égal d'acide sulfurique concentré a été ajouté par les côtés du tube à essai. La couche supérieure devient rouge et la couche d'acide sulfurique est de couleur jaune avec une fluorescence verte caractéristique de la présence de stéroïdes (Gibbs, 1974).

Détermination des terpénoïdes $\mathrm{A} 2 \mathrm{ml}$ d'infusé aqueux ont été ajoutés à $2 \mathrm{ml}$ d'anhydride acétique. L'apparition, lors de l'ajout d'acide sulfurique concentré $\left(\mathrm{H}_{2} \mathrm{SO}_{4}\right)$ le long des parois du tube, d'un anneau rouge sombre à l'interface indique la présence de terpénoïdes et la concentration en $\mathrm{H}_{2} \mathrm{SO}_{4}$ de cycles bleus et verts 168 indique la présence de terpénoïdes (Ayola, 2008).

Détermination des tanins Deux millilitres d'infusé ont été ajoutés à quelques gouttes d'acétate de plomb à $1 \%$. Un précipité jaunâtre indique la présence de tanins (Ardestani et Yazdamparast, 2007).

Détermination des saponines Cinq millilitres d'infusé aqueux ont été mélangés avec $20 \mathrm{ml}$ d'eau distillée puis agités dans un cylindre gradué pendant 15 minutes. Le gout amer et la présence de la mousse confirment l'existence de saponines (Kumar, 2009).

Détermination des anthocyanines Deux millilitres d'infusé aqueux sont ajoutés à $2 \mathrm{ml}$ d'acide chlorhydrique $2 \mathrm{~N}$ et d'ammoniaque. L'apparition d'une coloration rose-rouge devenant ensuite bleue-violette indique la présence d'anthocyanines (Paris, 1969).

Détermination des leuco-anthocyanidines Cinq millilitres d'infusé aqueux sont ajoutés à $5 \mathrm{ml}$ d'alcool isoamylique. L'apparition d'une couleur rouge au niveau de la couche supérieure indique la présence de leucoanthocyanines (Paris, 1969).

Détermination des coumarines Trois millilitres de $\mathrm{NaOH}$ à $10 \%$ ont été ajoutés à $2 \mathrm{ml}$ d'infusé aqueux. La 
couleur jaune obtenue indique la présence de coumarines (Rizk, 1982).

Détermination des émodines Deux millilitres de $\mathrm{NH}_{4} \mathrm{OH}$ et $3 \mathrm{ml}$ de benzène ont été ajoutés à $5 \mathrm{ml}$ d'infusé aqueux. L'apparition d'une couleur rouge indique la présence d'émodines (Rizk, 1982).

\section{Dosages des grandes classes chimiques}

Dosage spectrophotométrique des alcaloïdes Le dosage des alcaloïdes est fait selon la méthode montrée par Sreevidya et Yazdamparast (2003). Brièvement, après précipitation des alcaloïdes présents dans une prise d'essai de $5 \mathrm{ml}$ de solution d'extrait à $5 \mathrm{mg} / \mathrm{ml} 194$ par ajout de réactif de Dragendorff $\left(\mathrm{KBil}_{4}\right)$ et lavage du précipité à l'éthanol, le résidu est ensuite traité par $2 \mathrm{ml}$ de solution de sulfure disodique. Au précipité brun récupéré par centrifugation est ensuite ajoutée une solution d'acide nitrique. Un aliquot de $1 \mathrm{ml}$ de cette solution est prélevé et mélangé à $5 \mathrm{ml}$ de solution de thiourée pour mesure de l'absorbance à $435 \mathrm{~nm}$. La concentration en bismuth de la solution est calculée par comparaison avec les valeurs obtenues pour une courbe de calibration obtenue préalablement à partir de solution de nitrate de bismuth. Le complexe formé entre le bismuth et les alcaloïdes étant d'un ratio $1: 1$, la concentration en alcaloïdes de la solution d'extrait est donc déterminée.

Dosage des flavonoïdes Le dosage des flavonoïdes a été réalisé suivant la méthode colorimétrique montrée par beaucoup d'auteurs (Ardestani et Yazdamparast, 2007 ; Adida et al., 2016). Un aliquot de $500 \mu \mathrm{l}$ d'extrait aqueux à $1 \mathrm{mg} / \mathrm{ml}$ est mélangé avec $2 \mathrm{ml}$ d'eau distillée.

\section{RÉSULTATS}

Le choix des espèces ciblées dans l'analyse de la composition phytochimique est porté sur les plantes les plus couramment citées et ayant les meilleurs niveaux de fidélité c'est-à-dire suffisamment impliquées dans la catégorie HBD (Hygiène Bucco-Dentaire) (Tableau 1). Le nombre de personnes impliquées dans l'enquête est de cent vingt-cinq (125). Les plantes énumérées comme bâtonnets frotte-dents ou cure-dents dans la commune de Téssékéré sont au nombre de vingt-quatre (24). La plupart d'entre elles sont des arbustes (15) ou des arbres (8), seule une espèce d'herbacée a été citée (Waltheria indica). Elles se répartissent dans quinze (15) genres et neuf (9) familles (Tableau 1). La famille des Fabaceae comportant huit (8) espèces regroupées dans cinq (5) genres, est la plus évoquée. Elle renferme cinq (5) Fabaceae Mimosoideae (4 Acacia et un Dichrostachys), deux Fabaceae Caesalpinoideae (Bauhinia, Tamarindus) et une Fabaceae Faboideae (Dalbergia).
A cette solution sont ajoutés par étapes $150 \mu$ d'une solution de nitrite de sodium $\left(\mathrm{NaNO}_{2}\right)$ à $15 \%$ puis $150 \mu \mathrm{l}$ de chlorure d'aluminium $\left(\mathrm{AlCl}_{3}, 6 \mathrm{H}_{2} \mathrm{O}\right)$ à $10 \%$ et enfin 2 $\mathrm{ml}$ d'hydroxyde de sodium à $4 \%$ (Didi, 2020). Le volume final est complété à $5 \mathrm{ml}$ avec de l'eau distillée. Après incubation à $30^{\circ} \mathrm{C}$ pendant 20 minutes, l'absorbance de la solution est mesurée à $510 \mathrm{~nm}$ contre un blanc au spectromètre. La concentration en flavonoïdes de la solution est calculée par comparaison de la valeur mesurée avec une courbe de calibration obtenue préalablement à partir d'une solution de quercétine et les résultats sont exprimés en microgramme équivalent quercétine par milligramme de la matière végétale sèche ( $\mu \mathrm{g} \mathrm{CEQ} / \mathrm{mg}$ ).

Dosage des polyphénols Le dosage des polyphénols a été effectué suivant la technique décrite par Vermerris et Nicholson (2006). 0,1 ml de solution aqueuse d'extrait à $1 \mathrm{mg} / \mathrm{ml}$ sont mélangés avec $2 \mathrm{ml}$ d'une solution de carbonate de sodium à $2 \%$ nouvellement préparée, le tout est remué par un vortex. Après cinq minutes, $100 \mu \mathrm{l}$ du réactif de Folin-Ciocalteu (1 N) sont rajoutés au mélange, le tout est gardé pendant 30 minutes à température ambiante, et la lecture de l'absorbance est réalisée contre un blanc au moyen d'un spectophotomètre à $750 \mathrm{~nm}$. Une courbe d'étalonnage est faite simultanément dans des conditions similaires en considérant l'acide gallique comme contrôle positif (Fadili et al., 2015). Les résultats sont exprimés en microgramme équivalent acide gallique par milligramme de la matière végétale sèche ( $\mu \mathrm{g} \mathrm{GAE} / \mathrm{mg}$ ).

Le genre Acacia comprenant quatre (4) espèce (Acacia nilotica, Acacia senegal, Acacia seyal et Acacia tortilis) est le plus communément cité, les autres genres comptent chacune une espèce. Les autres familles à espèces multiples sont les Combretaceae (Anogeissus leiocarpus, Combretum aculeatum et Guiera senegalensis), les Capparaceae (Boscia senegalensis, Cadaba farinosa et Maerua crassifolia) et les Malvaceae (Grewia bicolor et Waltheria indica). Les dernières familles citées sont représentées chacune par une espèce. II existe cependant des familles (Balanitaceae et Anacardiaceae) qui ne comptent qu'une seule espèce chacune mais sont plus souvent citées que certaines familles à espèces multiples (Capparaceae et Malvaceae). La tige est la seule partie de la plante proposée dans l'hygiène buccodentaire (HBD). Cinq espèces: $M$. crassifolia, $S$. persica, $A$. senegal, $B$. rufescens, et $Z$. mauritiana sont exclusivement citées 
comme bâtonnet frotte dent. Leur niveau de fidélité est égal à cent (100) car n'ayant été proposées que dans l'hygiène bucco-dentaire. Toutes les autres plantes interviennent dans d'autres pratiques cosmétiques: tatouages buccaux, teinture des mains et des pieds autres que l'hygiène bucco-dentaire, ce qui explique le niveau de fidélité inférieur à cent. Les extraits aqueux ayant servi au dosage des polyphénols, flavonoïdes et alcaloïdes ont été préparés à partir des tiges des douze (12) plantes ainsi réparties en se basant sur l'indice de fidélité et le nombre de citation dans la catégorie (IP) (Diatta et al., 2019) : cinq (5) espèces avec un indice de fidélité maximal $(100 \%)$ trois espèces avec un indice élevé (entre 90\% - 98\%), une espèce dont l'indice est moyen $(58 \%)$ et trois à faible indice (entre $35 \%$ - 19\%) pour 3 espèces. (Tableau 1) (Diatta et al., 2019). 
Tableau 1 : Liste des espèces dont les tiges sont utilisées comme bâtonnets frotte-dents utilisées par les Peul de Widou Thiengoli (Téssékéré) (Diatta et al., 2019

\begin{tabular}{|l|l|l|l|l|l|l|}
\hline Nom Peulh & Espèce & Famille & Organe & Ip & lu & IF (\%) \\
\hline Gawdi & Acacia nilotica subsp astringents (Schumach. \& & Fabaceae & tige & 21 & 36 & 58,33 \\
\hline Thili & Acacia tortilis subsp raddiana Savi. & Fabaceae & tige & 29 & 119 & 24,36 \\
\hline Patuki & Acacia senegal (L.) Willd. & Fabaceae & tige & 87 & 87 & 100 \\
\hline Bulbi & Acacia seyal Del & Fabaceae & tige & 16 & 84 & 19,04 \\
\hline kodioli & Anogeissus leiocarpa (DC.) Guill. \& Perr & Combretaceae & tige & 05 & 14 & 35,71 \\
\hline Namari & Bauhinia rufescens Lam. & Fabaceae & tige & 15 & 15 & 100 \\
\hline Gissili & Boscia senegalensis (Pers.) Lam. ex Poir. & Capparaceae & tige & 8 & 8 & 100 \\
\hline Seing & Cadaba farinosa Forsk. & Capparaceae & tige & 01 & 1 & 100 \\
\hline Burli & Dichrostachys cinerea(L) Wight \& Arn & Fabaceae & tige & 01 & 1 & 100 \\
\hline Bakagni & Cissus populnea Guill. et Perr. & Vitaceae & tige & 01 & 1 & 100 \\
\hline Lawgnandi & Combretum aculeatum Vent & Combretaceae & tige & 01 & 1 & 100 \\
\hline Baddi & Commiphora africana (A.Rich.) Engl. & Burseraceae & tige & 85 & 86 & 98,83 \\
\hline Dialambani & Dalbergia melanoxilon Guill. \& Perr. & Fabaceae & tige & 01 & 1 & 100 \\
\hline Kelli & Grewia bicolor Juss. & Malvaceae & tige & 03 & 64 & 4,68 \\
\hline Géloki & Guiera senegalensis J. F. Gmel. & Combretaceae & tige & 14 & 14 & 100 \\
\hline Gial goti & Gymnosperia senegalensis (Lam.) Exell. & Celastraceae & tige & 01 & 1 & 100 \\
\hline Degeti & Maerua crassifolia Forks. & Capparaceae & tige & 13 & 13 & 100 \\
\hline Koyli & Mitragyna inermis Willd & Rubiaceae & tige & 04 & 12 & 33,33 \\
\hline Guddi & Salvadora persica L. & Salvadoraceae & tige & 34 & 34 & 100 \\
\hline Eeri & Sclerocarya birrea (A Rich.) Hochst & Anacardiaceae & tige & 84 & 86 & 97,67 \\
\hline Diadmi & Tamarindus indica L. & Fabaceae & tige & 02 & 2 \\
\hline Kafaki & Waltheria indica L. & Malvaceae & tige & 01 & 1 \\
\hline Diabi & Ziziphus mauritiana Lam. & Rhamnaceae & tige & 13 & 13 \\
\hline Muteki & Balanites aegyptiaca (L.) Delile & Zygophyllaceae & tige & 113 & 123 & 91,86 \\
\hline I : nombre & & 100 \\
\hline
\end{tabular}

Ip : nombre d'informateurs ayant affirmé l'emploi de l'espèce dans l'Hygiène Bucco-Dentaire (HBD) lu : nombre total d'informateurs ayant cités la plante dans n'importe quelle autre catégorie d'usage 
Analyse phytochimique

Composition phytochimique L'analyse de la composition phytochimique réalisée sur 10 plantes révèle une présence des tanins et des saponines chez toutes les espèces, contrairement aux leucoanthocyanines et terpénoides qui ne sont détectés dans presque aucune des plantes excepté $S$. persica (tableau 2). A leiocarpus et $S$ persica renferment six familles de composés sur huit et sont ainsi les plus riches en substances phytochimiques, suivis d'A. seyal et $G$. senegalensis avec cinq familles sur huit (Tableau 2). Les espèces qui renferment le moins de composés chimiques semblent toute appartenir au genre Acacia. II s'agit en effet d'A. nilotica qui ne comporte que trois classes chimiques (tanins, saponines et anthocyanines) sur les huit répertoriés, $A$. tortislis et $A$. senegal qui en comptent chacune trois 254 (tanin, saponine et coumarine voir tableau 2

Dosage des composés chimiques L'analyse phytochimique montre que la concentration en polyphénols, flavonoïdes et alcaloïdes varie d'une espèce à une autre (Tableau 3$)$. Quatre espèces ( $A$. leiocarpa, A. tortilis, A. seyal, A. nilotica) renferment toutes les familles chimiques (polyphénols, flavonoïdes et alcaloïdes) dosées alors que cinq (B. rufescens, $B$. aegyptiaca, C. africana, S. persica, S. birrea) n'en contiennent que deux (Tableau 3 ). Les autres espèces (Z. mauritiana, M. crassifolia et $A$. seyal) ne renferment que des flavonoïdes (Tableau 3). Les flavonoïdes constituent la famille chimique la plus fréquemment rencontrée et les trois (3) espèces qui en contiennent le plus sont respectivement $C$. africana $(536,84 \mu \mathrm{g} / \mathrm{mg}), A$. leiocarpa $(302,56 \mu \mathrm{g} / \mathrm{mg})$ et A. nilotica $(266,56 \mu \mathrm{g} / \mathrm{mg})$. (Tableau 3). Sept des extraits de bâtonnets frotte-dents dosés renferment des alcaloïdes alors que six contiennent des polyphénols. A. leiocapa se démarque par ses fortes teneurs en alcaloïdes $(46,36 \mu \mathrm{g} / \mathrm{mg})$, en polyphénols (348 $\mu \mathrm{g} / \mathrm{mg}$ ) (Tableau 3). A. nilotica est également riche en polyphénols $(283,5 \mu \mathrm{g} / \mathrm{mg})$ mais pauvre en alcaloïdes $(3,33 \mu \mathrm{g} / \mathrm{mg})$. Les concentrations en polyphénols sont largement supérieures à celles des alcaloïdes (Tableau 3) 
Diatta et al., J. Appl. Biosci. 2021 Diversité et composition phytochimique des bâtonnets frotte-dents (cure-dents) proposés chez les Peul de la commune de Tessékéré (Ferlo Nord, Sénégal)

Tableau 2 : Composition phytochimique de bâtonnets frotte-dents utilisés à Widou

\begin{tabular}{|c|c|c|c|c|c|c|c|c|}
\hline $\begin{array}{l}\text { Composés } \\
\text { Espèces }\end{array}$ & Stéroides & Terpenoides & Tanins & Saponines & Anthocyanines & Leucoanthocyanines & Coumarines & Emodines \\
\hline Sclerocarya birrea & - & - & + & + & + & - & + & - \\
\hline Salvadora persica & + & + & + & + & + & - & - & + \\
\hline Acacia nilotica & - & - & + & + & + & - & - & - \\
\hline Anogeissus leiocarpa & + & - & + & + & + & - & + & + \\
\hline Acacia seyal & + & - & + & + & + & - & + & - \\
\hline Acacia tortilis & - & - & + & + & - & - & + & - \\
\hline Acacia Senegal & - & - & + & + & - & - & + & - \\
\hline Ziziphus mauritiana & - & - & + & + & - & - & + & + \\
\hline Balanites aegyptiaca & - & - & + & + & - & - & + & + \\
\hline Guiera senegalensis & + & - & + & + & - & - & + & + \\
\hline
\end{tabular}

Tableau 3 : Teneur en alcaloïdes, flavonoïdes et polyphénols des extraits de bâtonnets frotte-dents

\begin{tabular}{l|l|l|l}
\hline Espèces & $\begin{array}{l}\text { Concentration en polyphénols } \\
(\mu \mathrm{gEAG} / \mathrm{mg})\end{array}$ & $\begin{array}{l}\text { Concentration en flavonoïdes } \\
(\mu \mathrm{gEQ} / \mathrm{mg})\end{array}$ & $\begin{array}{l}\text { Concentration en alcaloïdes } \\
(\mu \mathrm{g} / \mathrm{mg})\end{array}$ \\
\hline Acacia senegal & & 22,46 & \\
\hline Acacia nilotica & 283,5 & 266,56 & 3,33 \\
\hline Acacia seyal & 50,5 & 82,84 & 10,04 \\
\hline Acacia tortilis & 330 & 57,6 & 9,4 \\
\hline Anogeissus leiocarpa & 348 & 302,56 & 46,36 \\
\hline Balanites aegyptiaca & & 21,04 & 2,42 \\
\hline Bauhinia rufescens & & 191,42 & 8,16 \\
\hline Commiphora Africana & 181,5 & 536,84 & \\
\hline Maerua crassifolia & & 15,6 & \\
\hline Salvadora persica & & 2,46 & 6,66 \\
\hline Sclerocarya birrea & 200 & 191,22 & \\
\hline Ziziphus mauritiana & & 242 & \\
\hline
\end{tabular}

$\mu \mathrm{gEAG} / \mathrm{mg}$ : microgramme équivalent acide gallique par milligramme de la matière végétale sèche matière végétale sèche 


\section{DISCUSSION}

Les raison d'utilisation des espèces comme frotte-dent sont multiples et recouvrent des facteurs variés: écologiques (disponibilité de l'espèce végétale), pharmacologique (activité thérapeutique), organoleptiques (tendresse de la tige de l'espèce) et culturels (propriétés médico-magique et médicoreligieuses). L'usage de Balanites aegyptiaca (Mutorki) est très populaire. Toutefois, dans certaines localités de la commune, il est prohibé notamment en période d'hivernage. II semble que cette plante favoriserait la montée du venin en cas de morsure de serpent (Diatta et al., 2019). Commiphora africana (Baddi) est très apprécié car il procure une halène agréable à ceux qui l'utilisent comme bâtonnet frotte-dent, luttant ainsi contre la mauvaise haleine. La forte utilisation des bâtonnets de S. persica (Guddi) comme cure-dent par la population de la commune serait due à une raison religieuse (Diatta et al., 2019). Le cure-dent favori du prophète Mahomet PSL dans le désert aurait été les bâtonnets de la même espèce. D'autres croyances et représentations sociales ont été mentionnées au Ferlo et renvoient plus souvent à la chance (Z. mauritiana), à la volonté de garder le monopole d'une conversation ( $S$. birrea), à la santé et à la longévité ( $B$. aegyptiaca et $C$. africana) (Diatta et al., 2019). L'étude de l'usage des plantes comme bâtonnet frotte-dents dans l'Hygiène Bucco-Dentaire (HBD) permet d'identifier 24 plantes à Widou Thiengoli (commune de Tessékéré), soit à peu près la moitié de la richesse spécifique répertoriée par Arbonnier (2000) estimée à 40 espèces pour le même usage. Le bâtonnet frotte-dent permet d'éliminer la plaque dentaire par action mécanique (Ajay et al., 2011). Danielsen et al. (1989) précise qu'une utilisation de cinq (5) mn par jour suffit pour avoir des résultats satisfaisants. II revient toutefois sur le fait que cela ne fonctionne que lorsque l'usager consacre assez de temps au nettoyage dentaire. En effet, il est fréquent de repérer des usagers portant des bâtonnets frotte-dents à la bouche mais pas forcément concentrés sur le nettoyage dentaire. Au Ghana, environ 72 plantes interviennent dans ce sens (Blay, 2004). Les Anacardiaceae et les Combretaceae sont également des familles botaniques couramment utilisées au Bénin comme bâtonnets frotte-dents avec une espèce commune l'A. leiocarpa (Akpona et al., 2009). La prédominance au Ferlo des épineux (Fabaceae, Zygophyllaceae...), espèces xérophytes, ayant transformé leurs feuilles en épines pour limiter les pertes d'eau au Ferlo, renseigne sur les conditions difficiles du milieu. Ce phénomène se traduit par un comportement hydrique et photosynthétique, inhérent à la survie de ces espèces (Diouf et al., 2013). D'ailleurs, d'après Grouzis (1988), la nette dominance d'espèces telles que $B$. aegyptiaca, $A$. tortilis, $A$. senegal..., espèces utilisées comme bâtonnets frotte-dents, témoigne d'une bonne adaptation écologique aux sévères conditions d'aridité qui caractérisent le milieu. Le nombre d'espèces avec un indice de fidélité élevé et le fait que certaines n'interviennent qu'exclusivement dans l'hygiène bucco-dentaire traduisent la grande popularité de l'usage de ces plantes comme cure-dent dans la zone d'étude. Toutefois, il convient de signaler que le niveau de fidélité, pris isolément n'est pas forcément un indice de fiabilité sans failles ni limites. La preuve, une espèce peut avoir un très bon niveau de fidélité (100) alors qu'elle n'est citée qu'une seule fois (Cadaba farinosa, Waltheria indica...), comparée à d'autres espèces ( $A$. nilotica, $A$. seyal et $B$. aegyptiaca), certes impliquées dans d'autres catégories d'usage, mais plus communément citées dans l'hygiène buccodentaire voire tableau 1. B. aegyptiaca (Mutorki), espèce très répandue au Ferlo est la plante la plus fortement citée (113 informateurs). II semble en effet que l'usage de $B$. aegyptiaca soit lié à son abondance. D'ailleurs, omniprésent dans toute la zone sahélienne, fréquent au Sahara, $B$. aegyptiaca se rencontre depuis les côtes mauritaniennes jusqu'au Pakistan et l'Inde en Asie (Niang et al., 2014). 313 Selon Akpona et al. (2009), il y aurait une corrélation entre l'abondance d'une plante et son utilisation à des fins médicinales. $B$ aegyptiaca (Muteki) est suivi par A. senegal (Pattuki) ensuite puis, $S$. birrea (Eeri) toutes très répandues au Ferlo Nord Sénégal. Certaines des espèces recensées $(C$. africana et $S$. persica) n'existent presque plus au Ferlo en raison des contraintes anthropiques et écologiques. Commiphora africana, espèce aromatique fortement utilisée au Ferlo en raison de son parfum agréable pour lutter contre la mauvaise haleine détient la plus forte concentration en flavonoïdes $(536,84 \mu \mathrm{gEQ} / \mathrm{mg})$ est dans la catégorie des myrrhes (Commiphora. myrrha) (Su et al., 2011). Les résultats d' Ajay et al. (2011) relatifs à la comparaison de la brosse dentaire et du bâtonnet frotte-dent quant à l'élimination de la plaque dentaire ont montré que les deux méthodes se valaient. Cependant, d'autres études liées à l'évaluation de l'efficacité du bâtonnet frotte-dent comme outil d'hygiène orale soutiennent que le principe du bâtonnet est prolongé tant qu'il est en bouche [Almas, 1999 ; Darout et al., 2000 ; Mouhamed Ali et al., 2000 ; Baeshen et al., 2008). A. leiocarpa est aussi connue comme ayant une forte activité antibactérienne comme l'ont montré Taiwo et al. 
(1999) dans une étude visant à évaluer les propriétés bactéricides de dix plantes frotte-dents au Nigéria sur 25 bactéries différentes. L'extrait méthanolique de l'écorce de la tige d'A.senegal a montré un effet inhibiteur très modéré sur la croissance des micro-organismes parodontaux avec respectivement des concentrations minimales inhibitrices de $2,5 \mathrm{mg} / \mathrm{ml}$ chez Streptococcus mutans, $0,63 \mathrm{mg} / \mathrm{ml}$ chez Actinomyces viscus et $5 \mathrm{mg} / \mathrm{ml}$ chez Candida albicans (Khan et al., 2000). Nous pensons cependant qu'il aurait été plus pertinent d'utiliser à la place du méthanol (solvant toxique), un solvant aqueux plus similaire à la salive pour des résultats plus compatibles à la réalité et finalement les activités auraient peut-être été meilleures... Par ailleurs, de si fortes concentrations minimales inhibitrices traduisent une concentration considérable de l'extrait de la plante et non pas l'effet de la plante en tant que telle selon les valeurs limites proposées dans la littérature pour définir un extrait ou un composé actif (Cos et al., 2006 ; Gertsch, 2009). La limite proposée par Gertsch (2009) étant fixé à $50 \mu \mathrm{g} / \mathrm{mL}$ pour un extrait et $5 \mu \mathrm{M}$ pour constituant pur et une / Cl50 $100 \mu \mathrm{g} / \mathrm{mL}$ extrait et $25 \mu \mathrm{M}$ composé pur d'après Cos et al. (2006). S. persica communément appelé Miswak ou Siwak en Arabe est la plante au monde qui a le plus fait objet d'étude dans l'hygiène bucco-dentaire avec des propriétés bactéricides importantes sur une large gamme de souches bactériennes (Homer, 1990 ; Al Otabi et al., 2004). Toutefois, il convient de signaler que ces études font intervenir la racine comme matériel végétal alors qu'au Ferlo, c'est plutôt la tige qui est utilisée, potentiellement pour des raisons de conservation de la nature. Toutefois, il convient de signaler que les tiges sont rarement évoquées dans les usages, les feuilles étant plus communément utilisée par les populations dans d'autres pratiques (Guèye et al., 2012). La salvadorine, un alcaloïde présent dans $S$. persica, exerce une activité bactéricide, anti-inflammatoire et stimule la gencive (Malik et al., 1987 ; Deshpande et Kadam, 2013 ; Janot, 2013). Il a en effet été montré que les catéchines inactivent la toxine du vibrion cholérique et inhibent les glucosyltransférases bactériennes isolées chez Streptococcus mutans impliqué dans la survenue de la carie dentaire (Borris, 1996; Nakahara et Kabawata, 1996). Beaucoup de plantes utilisées comme bâtonnets frotte-dents ont des compositions phytochimiques qui ne sont toujours pas justifiées par les pharmaciens. L'intérêt des plantes sur les affections ou états bucco-dentaires a été mentionné pour quelques espèces impliquées dans notre étude. Ainsi, les écorces en mastication de Sclerocarya birrea ont été recommandées contre l'odontalgie et la carie dentaire ; la mastication des racines de Gymnosperia senegalensis contre l'odontalgie, la carie, la gingivite et la stomatite; les feuilles écrasées Boscia senegalensis en mastication contre la carie et les cure dent de Balanites aegyptiaca contre la carie (Diouf et al., 2011). Les composés végétaux constitueraient une des sources de substituts thérapeutiques les plus sûres et les plus précieuses sur les agents antimicrobiens (Deshpande et Kadam, 2013). Les teneurs en composés chimiques sont relativement élevées chez certaines espèces fortement utilisées dans la zone du Ferlo. C'est le cas d'A. leiocarpa, A. nilotica, $S$. birrea, $A$. tortilis et $C$. africana, riches en polyphénols ; A. nilotica, A. leiocarpa, $B$. rufescens, $C$. africana et $S$. birrea riches en flavonoïdes et $A$ leiocarpa et $A$ seyal riches en alcaloïdes. Parmi ces familles de nombreuses molécules ont été décrites pour leurs activités pharmacologiques variées (antibactérienne, anti-inflammatoire, analgésique, anesthésique, antiseptique, antiœdémateuse) (Houghstonet Rahman, 1998 ; Weller, 1998 ; Rohrer et Kunz, 2007). L'usage de ces espèces comme bâtonnets frotte-dents pourrait donc être justifié par la présence de composés actifs vis-à-vis de divers aspects des pathologies bucco-dentaires. Cependant cette mise en évidence sans évaluation de la teneur de certains phytoconstituants (tanin, stéroïdes, anthocyanines, leuco-anthocyanine, émodine, terpénoides, coumarines et émodine) renseigne peu sur l'efficacité ou non des bâtonnets frotte-dents quant à la prévention des pathologies bucco-dentaires. Une étude sur la quantification de ces phytoconstituants et l'activité antibactérienne des extraits de plante les plus riches permettrait de mieux orienter les populations vers les plantes les plus actives. Dans certaines spécialités comme la chirurgie, la parodontie, l'utilisation judicieuse des composés phytothérapiques permettrait d'éviter le recours aux antibiotiques et aux anti-inflammatoires de synthèse souvent onéreux pour le patient (Diouf et al., 2011). II existe en fait de nombreux exemples qui appuient la validité des phytoconstituants dérivés de plantes comestibles et médicinales en tant qu'agents antibactériens puissants (Houghston et Rahman, 1998). L'étude de Deshpande et Kadam (2013) sur l'analyse phytochimique et l'activité antibactérienne des écorces de la tige d'A. nilotica contre $S$. mutans confirme la présence des alcaloïdes, glucides, saponines, tanins, flavonoïdes. Cette étude a révélé une forte activité inhibitrice de l'écorce de tige d'Acacia nilotica, espèce dont les propriétés thérapeutiques sont reconnues au Ferlo. Cependant, il aurait été intéressant de montrer 
lequel de ces composés chimiques agit plus particulièrement contre le $S$. mutans et à quelle concentration pour une meilleure connaissance de la composition phytochimique de la plante. La forte activité antimicrobienne des tanins de $S$. persica et $A$. nilotica n'est plus à démontrer alors que les saponines de $B$. aegyptiaca ont une faible activité antimicrobienne (Van Wik et Van Oudtshoom, 2002 ; Runyoro et Gassapa, 2006). Les flavonoïdes sont rapportés pour leurs activités antibactériennes in vitro contre le vibrion cholérique, S. mutans, Shigella et d'autres bactéries (Borris, 1996; Nakahara et Kabawata, 1996). Ces

\section{CONCLUSION}

Si cette étude a permis de connaître la composition phytochimique et la teneur des grandes classes chimiques (polyphénols, flavonoïdes et alcaloïdes) des bâtonnets frotte-dent les plus communément exploités au Ferlo, elle a aussi montré la connaissance que les populations locales possédaient quant aux ressources thérapeutiques, culturelles et religieuses mises à leur disposition dans leur environnement. Elle permet ainsi de dévoiler une meilleure diversification dans le choix des bâtonnets frotte-dents, choix opéré en fonction des croyances culturelles et religieuses, des propriétés médicinales, de la tendreté ou non des fibres de l'espèce, du parfum de l'espèce... par comparaison aux pratiques bucco-dentaires modernes ayant pour vocations la prévention des caries, et paradontopathies, ainsi que la quête d'une bonne haleine. Nous demeurons

\section{BIBLIOGRAPHIE}

AbdEIRahman H F, Skaug N et Francis G W, (2002). In vitro antimicrobial effects of crude miswak extracts on oral pathogens in vitro, Saudi Dental Journal, 10(14): 15-21. 420

Adebajo AO, Adewumi CO. et Essein EE, 1983. Antiinfective agent of higher plants. International Symposium of Medicinal Plants 5th edition University of Ife, Nigeria, 152-158. 421

Adida H, Benariba N, Bechiri A, Chekroun E., et Djaziri $R, 2016$. Étude phytochimique et évaluation du pouvoir antiradicalaire des extraits de Pituranthos scoparius. Phytothérapie, 14(4): 207-212.

Ajay B, Sonal K, Sudhan S. et Jain M, 2011. Comparative effect of nemmstick and toothbrush on plaque removal and gingival health-A clinical trial. J Adv. Oral Research, 2(3): $51-56.422$ résultats sont compatibles avec ceux de Tenguria (2012). A. nilotica et $A$. leiocarpa font partie des plantes à forte concentration tannifère (12 à 32\%) (Sereme et al., 2008). D'après le même auteur, les extraits de racines d'A. nilotica très riches en tanins sont utilisés contre les maux de dents. De nombreuses plantes contenant des alcaloïdes, des tanins, des glycosides possèdent une activité antimicrobienne contre de nombreux microorganismes, comme l'a montré Adebajo et al. (1983). Ces résultats sont en corrélation avec ceux de Banso (2009) sur l'activité antimicrobienne de l'écorce de la tige d'A. nilotica sur divers microorganismes

toutefois conscients que toutes molécules répertoriées ne présenteront pas forcément une activité, que tout dépend aussi de la concentration de la molécule active dans l'extrait / en contact avec les pathogènes lors de l'usage, que les mesures d'activités antimicrobiennes vis-à-vis des souches pathogènes parodontaux permettent d'avancer avec plus de certitude quant à la validité de l'usage.

Remerciements : Ce travail a bénéficié du financement du Labex DRIIHM de I'OHMi Tessekere et de la foundation Veolia que nous remercions sincèrement pour tant d'appui. Ce travail a bénéficié d'une aide de l'État gérée par l'Agence Nationale de la Recherche au titre du Labex DRIIHM, programme « Investissements d'avenir » portant la référence ANR-11-LABX-0010

Akpona HA., Akpona JDT., Awokou SK., Yemoa A et Dossa LOSN, 2009. Inventory, folk classification and pharmacological properties of plant species used as chewing stick in Benin Republic. Journal of Medicinal Plants Research, 3(5): 382-389.

Al-LafiT AH et Ababneh H, 1995. The effect of the extract of the miswak (chewing stick) used in Jordan and the middle East on oral bacteria », International Dental Journal, 45(3):218-222. Almas K., 1999. The antimicrobial effects of extracts of Azadirachta indica (Neem) and Salvadora persica (Arak) chewing sticks. Indian journal of dental research: 10(1): 23- 26.

Al-Otaibi M, Al-Harthy M, Gustafsson A, Johansson A, Claesson R et Angmar- Månsson B, 2004. Subgingival plaque microbiota in Saudi Arabians after use of miswak chewing stick and 
toothbrush. Journal of Clinical Periodontology, 31(12) : 1048-1053

Arbonnier M, 2000. Arbres, Arbustes et Lianes des Zones Sèches d'Afrique de l'Ouest. Ed. CIRAD-MNHN-UICN. Paris : 549-557.

Ancey V, Ickowicz A, Corniaux C, Manoli C, Magnani S, 2009. Stratégies pastorales de sécurisation chez les Peuls du Ferlo (Sénégal), Journal des africanistes, 78(1-2): 105-119.

Ardestani A. et Yazdanparast R, 2007. Inhibitory effects of ethyl acetate extract of Teucrium polium on in vitro protein glycoxidation. Food and chemical toxicology, 45(12) : 2402- 2411.

Aubréville, A. (1949). Climats, forêts et désertification de I'Afrique tropicale.

Ayoola GA, Coker HA, Adesegun SA, Adepoju-Bello AA, Obaweya K, Ezennia EC et Atangbayila TO, 2008. Phytochemical screening and antioxidant activities of some selected medicinal plants used for malaria therapy in Southwestern Nigeria. Tropical Journal of Pharmaceutical Research, 7(3):1019-1024.

Baeshen HA. Kjellberg H., Lingström P et Birkhed D, 2008. Uptake and release of fluoride from fluoride-impregnated chewing sticks (miswaks) in vitro and in vivo. Caries research, 42(5): 363-368.

Bakhoum A., 2013. Dynamique des ressources fourragères : Indicateur de résilience des parcours communautaires de Téssékéré au Ferlo (Nord Sénégal). Thèse doct., Ecologie, UCAD.

Banso A, 2009. Phytochemical and antibacterial investigation of bark extract of Acacia nilotica. J. Med.Plants Res, 3(2): 082-085.

Begossi A, 1996. Use of ecological methods in ethnobotany: Diversity indices. Ecological Methods in Ethnobotany, 50(3) : 280-289.

Berhaut J, 1967. Flore du Sénégal plus complète avec les forêts humides de la Casamance. Dakar, ClairAfrique: $485 p$.

Blay D, 2004. Dental hygiene and livelihoods: a case of chewing sticks in Ghana. Forest products, livelihoods and conservation: case studies of non-timber forest product systems, 2: 25-36.

Borris R. P., 1996. Natural product research: perspective from a major pharmaceutical company, Journal of ethnopharmacology, 51(1-3): 29-38.

CSE (Centre de Suivi Écologique) (2007), Rapport technique 2006, janvier 2007, 49 p. 437 Cos $P$, Vlietinck AJ, Berghe D, 2006. Anti-infective potential of natural products: How to develop a stronger in vitro "proof-of-concept." Journal of Ethnopharmacology, 106(3): 290-302.

Danielsen BO, Baelum V, Manji F et Fejerskov O, (1989). Chewing sticks, toothpaste, and plaque removal. Acta Odontologica Scandinavica, 47(2) : 121-125.

Darout IA., Albandar JM. et Skaug N, 2000. Periodontal status of adult Sudanese habitual user of miswak, toothbrushes. Acta Odontologica Scandinavica, 58(1): 25-30.

Deshpande SN. et Kadam DG, 2013. Phytochemical analysis and antibacterial activity of Acacia nilotica against Streptococcus mutans. International Journal of Pharmacy and Pharmaceutical Sciences, 5(1): 236-238.

Diallo A., Agbangba EC, Thiaw A et Guissé A, 2012. Structure des populations d'Acacia senegal (L.) Willd dans la zone de Tessékéré (Ferlo nord), Sénégal. Journal of Applied Biosciences 59: 4366-4374.

Didi M, 2020. Evaluation of the antioxidant activity of the coffee parchment. Scientific Study and Research.

Diouf M, Cisse D. et Toure B, 2011. Plantes médicinales au Sénégal et affections buccodentaires : utilisations et intérêts, Santé tropicale, vol $34 n^{\circ}$ 3.

Diouf M, Boëtsch G, Cissé D, Tal-Dia A et Bonfil JJ, 2013. Modes de vie et santé buccodentaire chez les populations Peulhs du Ferlo au Sénégal. Revue d'Épidémiologie et de Santé Publique, 62, S217, 22: 187-192.

Gertsch J, 2009. How scientific is the science in ethnopharmacology? Historical perspectives and epistemological problems. Journal of Ethnopharmacology, 122(2), 177-183.

Gibbs R. D., 1974. Chemotaxonomy of Flowering Plants (4 Volumes). McGill-Queen's Press-MQUP. Montréal

Grouzis M, 1988. Structure, productivité et dynamique des systèmes écologiques sahéliens (Mare d'Oursi, Burkina Faso). Thèse de doctorat en Sciences Naturelles, ORSTOM, Paris.

Guèye M, Cisse A, Diatta CD., Diop S et Koma S, 2012. Étude ethnobotanique des plantes utilisées contre la constipation chez les Malinké de la communauté rurale de Tomboronkoto. Int.J.Biol.Chem. Sci 6(2) : 778-779.

Guissé A., 2013. "Portraits d'arbres" in Boetsch G., Guerci A., Guèye L., Guissé A (Ed) La Grande 
Muraille Verte. Toulouse Privat, pp. 64-98. 449 Guibert B, 2008. Restrictions et reconstructions successives d'un espace vital (pastoral, social et politique) des Peuls du Ferlo sénégalais durant la deuxième moitié du XXe siècle, PAPF, Saint Louis, 26 p.

Heinrich M, Ankli A, Frei B, Weimann C. et Sticher O, 1998. Medicinal plants in Mexico: Healers' consensus and cultural importance. Social Science \& Medicine, 47(11): 1859- 1871.

Homer DAKMF, 1990. Inhibition of protease activities of periodontopatihic bacteria by extracts of plants used in Kenya as Ghewing sticks (Miswak) » Arch Oral Biol 35: 421-424.

Houghton P et Raman A, 1998. Laboratory Handbook for the Fractionation of Natural Extracts. VI vol: $199 p$.

Hunn ES, 2002. Evidence for the precocious acquisition of plant knowledge by Zapotec children. Ethnobiology and biocultural diversity, 604, 13.

Janot C, 2013. Intérêt clinique de l'usage des bâtonnets frotte dent Analyse physique et chimique de bois en provenance de Burkina Faso. Thèse doct., chirurgie dentaire.

Khan MNNO, Olipa N et Mecky IN, 2000. Antimicrobial activity or Tanzanian chewing sticks against oral pathogenic microbes. Pharmaceutical Biology 38(3): 235- 240.

Kumar A, llavarasan R, Jayachandran T, Decaraman M, Aravindhan $\mathrm{P}$, Padmanabhan $\mathrm{N}$ et Krishnan $\mathrm{M}$ $\mathrm{RV}, 2009$. Phytochemicals investigation on a tropical plant, Syzygium 171 cumini from Kattuppalayam, Erode district, Tamil Nadu, South India. Pakistan Journal of Nutrition, 8(1): 83-85.

Lewis WH, 1980. Plants used as chewing sticks. Journal of Preventive Dentistry, 6: 71-73

Malik S, Ahmad SS, Haider S L, 1987. Salvadocirine: A new indole alkaloi from leaves of Salvadora persica. Tetrahedron Lett., 28: 163-164.

Mishra AK, Mishra A, Bhargava A, Pandey AK, 2008. Antimicrobial activity of essential oils from the leaves of Cinnamomum spp. National Academy Science Letters, 31(11-12): 341-345.

Mouhamed-Ali K, 2002. Le batonnet frotte-dent : aspect scientifique et religieux. Thèse de chirurgie dentaire, Paris.

Nakahara K et Kabawata N, 1996. Inhibitory effect of oolong tea polyphenols and glucosyltransferases of mutans streptococci,
Applied and Environment Microbiology, 59(4): 968-73 193.

Niang K, Ndiaye O, Diallo A et Guisse A, 2014. Flore et structure de la végétation ligneuse le long de la Grande Muraille Verte au Ferlo, nord Sénégal. Journal of Applied Biosciences, 79(1) : 69386946.

Ohmagari $\mathrm{K}$ et Berkes F, 1997. Transmission of indigenous knowledge and bush skills among the Western James Bay Cree women of subarctic Canada. Human Ecology, 25(2) : 197222

Paris R, 1969. Precis de matiere medicinale. Collection de précis de pharmacie. Paris : Masson

Pasquini A, 2013. Pour Offrir un sourire... Evolution de l'hygiène bucco-dentaire au fil des siècles et des civilisations. Edition universitaires européennes 340 pages

Rizk A, 1982. Constituents of Plants Growing in Qatar. Fitoterapia, 52(2) : 35-42.

Rohrer U, Kunz E M, Lenkeit, K, Schaffner, W, et Meyer, $\mathrm{J}, 2007$. Antimicrobial activity of Mahonia aquifolium and two of its alkaloids against oral bacteria. Revue mensuelle suisse d'odontostomatologie, 117(11), 1126-1131.

Rotimi VO, Laughon BE, Bartlett JG. et Mosadomi HA, 1988. Activities of Nigerian chewing stick extracts against Bacteroides gingivalis and Bacteroides melaninogenicus. Antimicrobial agents and Chemotherapy, 32(4): 598-600.

Runyoro DKB, Ngassapa OD, Matee MIN, Joseph CC. et Moshi MJ, 2006. Medicinal plants used by Tanzanian traditional healers in the management of Candida infections. Journal of Ethnopharmacology, 106(2) : 158-165.

Sereme A, Milogo-Rasolodimby J, Guinko S. et Nacro M, 2011. Propriétés thérapeutiques des plantes à tanins du Burkina Faso. Pharmacopée et médecine traditionnelle africaine, 15: 41- 49.

Su S, Wang T, Duan JA, Zhou W, Hua Y Q, Tang YP., ...et Qian DW. (2011). Anti-inflammatory and analgesic activity of different extracts of Commiphora myrrha. Journal of ethnopharmacology, 134(2), 251-258.

Sreevidya N et Mehrotra S, 2003. Spectrophotometric method for estimation of alkaloids precipitable with Dragendorff's reagent in plant materials. Journal of AOAC international, 86(6): 11241127. 
Taiwo O, Xu HX. et Lee SF, 1999. Antibacterial activities of extracts from Nigerian chewing sticks. Phytotherapy Research, 13(8): 675-679.

Tenguria MCP, 2012. Estimation of total polyphenolic content in aqueous and methanolic extract from the bark of Acacia nilotica Int. J. Pharmacol.science Res, 3(9): 3458-3461.

Van Wyk B E, Heerden F V, et Oudtshoorn B V, 2002. Poisonous plants of South Africa. Pretoria, Briza Publications, 288 pages

Vermerris W et Nicholson R., 2006. Isolation and identify cation of phenolic compounds - A practical guide. In W. Vermerris \& R. Nicholson (Eds.), Phenolic Compound Biochemistry (pp. 151196). Dordrecht, Netherlands: Springer.

Weckerle, CS, De Boer HJ, Puri, RK., Van Andel T, Bussmann, R W et Leonti $M, 2018$. Recommended standards for conducting and reporting ethnopharmacological field studies. Journal of Ethnopharmacology, 210: 125-132. 478 Weller S C R A (1998) Systematic Data collection. Qualitative Research Methods. USA.

Zarger RK, 2002. Acquisition and transmission of subsistence knowledge by Q'eqchi'Maya in Belize. Ethnobiology and biocultural diversity, 592-603.

Zhishen J, Mengcheng T et Jianming W, 1999. The determination of flavonoid contents in mulberry and their scavenging effects on superoxide radicals. Food chemistry, 64(4) : 555-559. 